\title{
Test-retest reliability of the 'Reading the Mind in the Eyes' test: a one-year follow-up study
}

\author{
Enrique G Fernández-Abascal ${ }^{1}$, Rosario Cabello ${ }^{2}$, Pablo Fernández-Berrocal ${ }^{3 *}$ and Simon Baron-Cohen ${ }^{4}$
}

\begin{abstract}
Background: The 'Reading the Mind in the Eyes' (Eyes) test is an advanced test of theory of mind. It is widely used to assess individual differences in social cognition and emotion recognition across different groups and cultures. The present study examined distributions of responses and scores on a Spanish version of the test in a non-clinical Spanish adult population, and assessed test-retest reliability over a 1-year interval.

Methods: A total of 358 undergraduates of both sexes, age 18 to 65 years, completed the Spanish version of the test twice over an interval of 1 year. The Bland-Altman method was used to calculate test-retest reliability.

Results: Distributions of responses and scores were optimal. Test-retest reliability for total score on the Eyes test was $.63(P<.01)$, based on the intraclass correlation coefficient. Test-retest reliability using the Bland-Altman method was fairly good.

Conclusions: This is the first study providing evidence that the Eyes test is reliable and stable over a 1-year period, in a non-clinical sample of adults.
\end{abstract}

Keywords: Reading the mind in the eyes, Reliability, Assessment, Social cognition, Theory of mind

\section{Background}

Psychology researchers have developed reliable instruments for evaluating social cognition and emotional and social processing in both the laboratory and the clinic [1]. Social cognitive studies examine how people process information in the social environment, particularly perceiving, interpreting, and responding to the mental states (intentions, feelings, perception, and beliefs), dispositions, and behaviors of others [2-5]. These processes are tightly linked to processes referred to as emotion recognition and 'theory of mind', that allow individuals to imagine the mental state of others [6] to both predict their behavior and respond appropriately. Numerous studies have shown that deficits in emotion recognition and theory of mind compromise social interaction and are related to conditions such as schizophrenia $[7,8]$, autism [9-11], eating disorders [12-14], bipolar disorder $[15,16]$, social anxiety [17], and borderline personality disorder [18].

\footnotetext{
* Correspondence: berrocal@uma.es

${ }^{3}$ Department of Basic Psychology, Faculty of Psychology, University of

Malaga, Campus Teatinos, s/n, 29071, Malaga, Spain

Full list of author information is available at the end of the article
}

Different instruments have been developed to assess deficits in social cognition in adults. Instruments designed to assess emotion recognition require the individual to identify emotions and their intensity on the basis of different stimuli, such as facial expressions in the 'facial emotion identification task' [19], spoken phrases in the 'Reading the Mind in the Voice' test [20] or computer-generated, distorted facial pictures (morphing) [21]. Instruments to assess theory of mind, in contrast, often require individuals to read short stories and answer questions about them [22]. These instruments are intended to assess theory of mind in individuals with autism or Asperger Syndrome, but may also be applicable to other conditions [23].

To provide more detailed information about theory of mind dysfunction, Baron-Cohen et al. developed the 'Reading the Mind in the Eyes' test, an advanced test of theory of mind [23]. The first version consisted of 25 photographs of actors and actresses showing the facial region around the eyes. The participant is asked to choose which of two words best describes what the person in the photograph is thinking or feeling. These words refer to both basic mental states (for example,

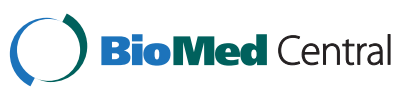


'happy') and complex mental states (for example, 'arrogant') [23]. In this way, the Eyes test aimed to evaluate social cognition in adults by assessing their ability to recognize the mental state of others using just the expressions around the eyes, which are key in determining mental states [24].

The original 'Reading the Mind in the Eyes' test had some limitations because the number of items and the binomial response format did not sufficiently differentiate individuals receiving higher scores. Thus a revised 'Reading the Mind in the Eyes' test was created, in which the number of items was increased to 36 and the number of possible responses (single-word descriptors of possible mental states) was increased to 4 , reducing the maximum correct guess rate to $25 \%$ [1]. The possible mental state descriptors refer mostly to complex mental states. This advanced test was designed to have sufficient analytical complexity to be appropriate for adults with and without psychopathology, brain damage or dementia, to assess factors that might contribute to social difficulties. In this way, the test is intended to allow assessment of social cognition in an adult population with average intelligence.

Although conceived as an advanced theory of mind test [1], the Eyes test is also used to assess emotion recognition. Completing the instrument requires not only the ability to recognize emotional expressions but also the ability to determine the complex cognitive mental state of an individual based on a partial facial expression. Together, these abilities presuppose that the individual possesses a mental state lexicon and knows the meaning of mental state terms [1].

Studies of social cognition impairments in clinical populations show that typical individuals score significantly higher on the Eyes test than do individuals with schizophrenia $[7,8]$, autism $[9,10]$, eating disorders $[12,13,25]$, and social anxiety [17] (for a review, see [26]). These studies indicate that the Eyes test is reliable for assessing social cognition in adults. The Eyes test has also proven useful for assessing social intelligence and its subtle impairment in different cultures, as shown in studies using translations of the Eyes test into Turkish, Hungarian, Japanese, French, German, and Argentinian Spanish [7,27-31].

Most studies with the Eyes test have not reported information on test-retest reliability [26]. This is essential because the Eyes test, like tests explicitly designed to test emotion recognition [32], has psychometric properties that prevent straightforward calculation of Cronbach's alpha. Calculating this parameter is complex because researchers are limited to comparing the number of correct responses between individuals. Thus, many studies involving the Eyes test do not include Cronbach's alpha, making it impossible to draw reliable intergroup comparisons, such as comparisons between clinical and control groups or comparisons between the same group before and after an intervention. Intergroup comparisons are also important for cross-cultural studies, which aim to test if cultures differ more in how they identify complex mental states than simpler mental states [26]. Such studies are important for indicating whether the Eyes test should be adapted specifically for different cultures.

Recent studies have addressed this gap by reporting acceptable test-retest stability for the adult version of the Eyes test [26,33] as well as for the child version [34]. The time intervals for retesting in these studies were relatively short, ranging from 2 weeks to 1 month. In order to provide the first assessment of long-term testretest reliability of the Eyes test, as well as the first detailed validation of the test in a Spanish population, the present study (1) examined the distribution of responses and scores on a Spanish version of the Eyes test in a nonclinical Spanish population, and (2) assessed the 1 -year test-retest reliability.

\section{Method \\ Participants}

A total of 358 first-year psychology undergraduates enrolled at the Universidad Nacional de Educación a Distancia (UNED, Spain) took part. The sample comprised 75 men and 283 women, with a mean age at the first testing of 34.23 years (sd, 9.02; range, 18 to 65). This bias toward female participants simply reflects the sex ratio in those who choose to study psychology at the undergraduate level. All participants were volunteers who gave written informed consent and who received personalized reports of results at the end of the study. The study was carried out in accordance with the Declaration of Helsinki. Ethics approval was obtained from the Research Ethics Committee, UNED.

\section{Procedure}

The first testing took place during May and June 2011; the second testing took place during the same months in 2012. During both testing sessions, the survey was administered using a computer program that recorded identification data for each participant, displayed test items and saved the responses.

\section{Measures}

The revised Eyes test [1] was used to generate a Spanish version of the Eyes test. Two translators, both with PhDs in psychology and experts in cognition and emotion, created a Spanish version of the instrument, which was then back-translated into English by two independent translators. In this version, as in the English-language original, participants were shown 36 photographs of eye regions of individuals and asked, for each photograph, to 
choose one of four possible words to describe the mental state of the person shown. One point was assigned for each correct response, so scores could range from 0 to 36. This Spanish version is available from the authors on request.

\section{Statistical analysis}

Data were analyzed using the Statistical Package for the Social Sciences (SPSS), version 19.0. (Armonk, NY: IBM Corp). The Bland-Altman plot to compare test and retest results was calculated using the MedCalc program, version 12.3 (MedCalc ${ }^{\mathrm{Tw}}$, Mariakerke, Belgium, http:// www.medcalc.be). All tests were two-tailed and were conducted at the $5 \%$ level of statistical significance.

\section{Results}

Table 1 shows the correct answer for each item on the Spanish version of the Eyes test, and the percentages of participants that selected each answer on the test and retest.

Nearly all items on the test were answered correctly by more than $50 \%$ of participants. The only exception was item 19 , which was answered correctly by only $39.0 \%$ of respondents during the test and by only $40.4 \%$ during the retest. The next most frequently selected answer B was chosen by $36.2 \%$ and $34.4 \%$ of respondents during testing and retesting, respectively. The mean percentage of items correctly answered was $75.51 \%$ on the test and $75.46 \%$ on the retest. There were no significant differences in the percentages of respondents choosing the correct answer across all items during testing and retesting ( $\mathrm{t}=.093, P<.926)$. In fact, for all items, the correct answer was chosen far more often than the next most frequently selected option.

Converting the mean percentages above to the 0 to 36 scale of the Eyes test gave mean point scores of 27.18 $(\mathrm{sd}=3.59)$ on the test and 27.24 $(\mathrm{sd}=3.67)$ on the retest $(\mathrm{t}=.36, P<.722)$. Test-retest stability was assessed using the intraclass correlation coefficient (ICC), which was .63 for the total score $(\mathrm{P}<.01)$. Table 2 shows test-retest correlations for each of the items. Correlations for all items except item 18 were positive and significant. Although results for item 18 showed 330 of 358 possible coincidences, no significant linear correlation was found. This result does not mean that test and retest results for item 18 were independent, but rather that they were not linearly related. The Bland-Altman plot [35] was used to examine test-retest concordance. This graphical approach allows for the examination of the agreement between repeated measurements by plotting the differences between test and retest scores against the mean value of the test and retest scores for each participant. Confidence intervals for the mean difference are calcu-
Table 1 Spanish version of the 'Reading the Mind in the Eyes' test

\begin{tabular}{|c|c|c|c|c|c|c|c|c|}
\hline \multirow[t]{2}{*}{ Item } & \multicolumn{2}{|c|}{ Answer $A$} & \multicolumn{2}{|c|}{ Answer $B$} & \multicolumn{2}{|c|}{ Answer C } & \multicolumn{2}{|c|}{ Answer D } \\
\hline & Test & Retest & Test & Retest & Test & Retest & Test & Retest \\
\hline 1 & 66.9 & 57.1 & 17.8 & 21.4 & 12.5 & 17.3 & 2.8 & 3.6 \\
\hline 2 & 13.6 & 10.9 & 63.8 & 62.1 & 2.2 & 2.8 & 20.3 & 24.0 \\
\hline 3 & 1.1 & 1.4 & 4.5 & 5.6 & 75.2 & 71.0 & 19.2 & 21.4 \\
\hline 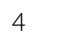 & 0.6 & 2.2 & 81.1 & 83.8 & 0.8 & 0.6 & 17.0 & 12.0 \\
\hline 5 & 2.5 & 2.8 & 4.7 & 5.0 & 92.5 & 91.6 & 0.3 & 0.3 \\
\hline 6 & 1.7 & 2.5 & 75.2 & 79.9 & 18.9 & 14.8 & 4.2 & 2.5 \\
\hline 7 & 6.1 & 5.3 & 21.7 & 16.2 & 64.6 & 71.3 & 7.2 & 7.0 \\
\hline 8 & 88.0 & 88.3 & 4.2 & 7.2 & 5.0 & 3.3 & 2.8 & 0.8 \\
\hline 9 & 4.5 & 6.7 & 11.4 & 10.6 & 2.2 & 3.3 & 81.9 & 78.8 \\
\hline 10 & 71.0 & 73.0 & 21.4 & 20.9 & 5.6 & 3.6 & 1.7 & 1.9 \\
\hline 11 & 4.2 & 3.3 & 3.9 & 5.8 & 74.1 & 72.1 & 17.8 & 18.1 \\
\hline 12 & 12.5 & 12.8 & 2.2 & 3.6 & 80.8 & 79.7 & 4.5 & 3.3 \\
\hline 13 & 4.5 & 4.2 & 80.8 & 81.6 & 1.7 & 1.7 & 13.1 & 12.0 \\
\hline 14 & 6.7 & 8.9 & 4.2 & 3.3 & 0.3 & 0.3 & 88.9 & 86.9 \\
\hline 15 & 86.9 & 88.6 & 9.2 & 7.5 & 1.1 & 1.7 & 2.5 & 1.7 \\
\hline 16 & 0.6 & 1.7 & 85.8 & 83.6 & 1.4 & 2.2 & 12.0 & 12.3 \\
\hline 17 & 54.3 & 57.1 & 27.3 & 27.9 & 1.7 & 1.1 & 16.7 & 13.4 \\
\hline 18 & 96.4 & 94.7 & 1.7 & 3.6 & 0.3 & 0.6 & 1.7 & 0.8 \\
\hline 19 & 12.8 & 9.5 & 36.2 & 4 & 12.0 & 11.1 & 39.0 & 40.4 \\
\hline 20 & 5.8 & 4.7 & 89.4 & 89.7 & 4.7 & 5.3 & 0.0 & 0.0 \\
\hline 21 & 9.5 & 8.1 & 75.2 & 78.0 & 13.9 & 13.4 & 1.1 & 0.3 \\
\hline 22 & 70.8 & 74.1 & 0.8 & 0.6 & 11.7 & 9.5 & 16.4 & 15.6 \\
\hline 23 & 4.5 & 3.6 & 5.8 & 6.4 & 65.5 & 69.4 & 24.2 & 20.3 \\
\hline 24 & 73.5 & 69.1 & 8.4 & 12.0 & 1.9 & 2.5 & 16 & 15.9 \\
\hline 25 & 2.8 & 3.9 & 18.7 & 18.9 & 8.1 & 7.2 & 70.5 & 69.4 \\
\hline 26 & 6.4 & 5.0 & 6.7 & 7.5 & 75.2 & 76.9 & 11.4 & 10.3 \\
\hline 27 & 0.3 & 0.3 & 64.1 & 65.2 & 24.5 & 20.9 & 11.1 & 13.4 \\
\hline 28 & 83.6 & 82.5 & 1.1 & 0.8 & 3.3 & 5.6 & 11.4 & 10.6 \\
\hline 29 & 9.2 & 3.6 & 4.5 & 4.7 & 5.3 & 5.0 & 81.1 & 86.4 \\
\hline 30 & 3.9 & 3.3 & 88.6 & 86.4 & 4.7 & 7.5 & 2.8 & 2.5 \\
\hline 31 & 10.9 & 10.69 & 57.1 & 55.2 & 7.5 & 8.1 & 24.2 & 25.6 \\
\hline 32 & 78.0 & 74.4 & 4.5 & 4.7 & 5.8 & 9.2 & 11.4 & 11.4 \\
\hline 33 & 9.5 & 10.0 & 25.1 & 21.7 & 4.2 & 5.3 & 61.3 & 62.4 \\
\hline 34 & 9.2 & 12.5 & 13.6 & 14.2 & 72.1 & 68.8 & 4.5 & 4.2 \\
\hline 35 & 13.9 & 11.7 & 77.7 & 77.7 & 5.0 & 7.2 & 3.1 & 3.1 \\
\hline 36 & 0.8 & 0.8 & 1.4 & 1.1 & 87.5 & 89.4 & 10.0 & 8.4 \\
\hline
\end{tabular}

Distribution of responses in percentages for test and retest assessment $(n=358)$. 'Correct' responses according to original study are in bold.

lated to determine if the mean difference deviates significantly from zero (Figure 1).

The mean difference between test and retest responses across all participants was $-0.06(S D=3.12)$, indicating no significant change in results between testing and 
Table 2 Spearman's Rho correlations between test and retest for each item for right and wrong answers ( $n=358)$

\begin{tabular}{|c|c|}
\hline Item & Spearman's Rho correlation \\
\hline 1 & $.391^{* *}$ \\
\hline 2 & $.297^{* *}$ \\
\hline 3 & $.278^{* *}$ \\
\hline 4 & $.197^{* *}$ \\
\hline 5 & $.226^{* *}$ \\
\hline 6 & $204^{* *}$ \\
\hline 7 & $.144^{* *}$ \\
\hline 8 & $.151^{* *}$ \\
\hline 9 & $.390^{* *}$ \\
\hline 10 & $.323^{* *}$ \\
\hline 11 & $.248^{* *}$ \\
\hline 12 & $.183^{* *}$ \\
\hline 13 & $.253^{* *}$ \\
\hline 14 & $.276^{* *}$ \\
\hline 15 & $.159^{* *}$ \\
\hline 16 & $.274^{* *}$ \\
\hline 17 & $.252^{* *}$ \\
\hline 18 & .095 \\
\hline 19 & $.201^{* *}$ \\
\hline 20 & $.110^{*}$ \\
\hline 21 & $.192^{* *}$ \\
\hline 22 & $.278^{* *}$ \\
\hline 23 & $.189^{* *}$ \\
\hline 24 & $.277^{* *}$ \\
\hline 25 & $.289^{* *}$ \\
\hline 26 & $.129^{*}$ \\
\hline 27 & $.179^{* *}$ \\
\hline 28 & $.197^{* *}$ \\
\hline 29 & $.207^{* *}$ \\
\hline 30 & $.190^{* *}$ \\
\hline 31 & $.124^{*}$ \\
\hline 32 & $.306^{* *}$ \\
\hline 33 & $.231^{* *}$ \\
\hline 34 & $.162^{* *}$ \\
\hline 35 & $.184^{* *}$ \\
\hline 36 & $.115^{*}$ \\
\hline
\end{tabular}

${ }^{*} P<.05,{ }^{* * P}<.01$.

retesting one year later. The $95 \%$ confidence interval (CI) for the mean difference was -6.17 to 6.05 ; thus, the CI included 0. Most results fell within the 95\% CI, and those that did not failed to show any tendency, suggesting that they reflected chance variation. Estimated measurement error based on within-subject

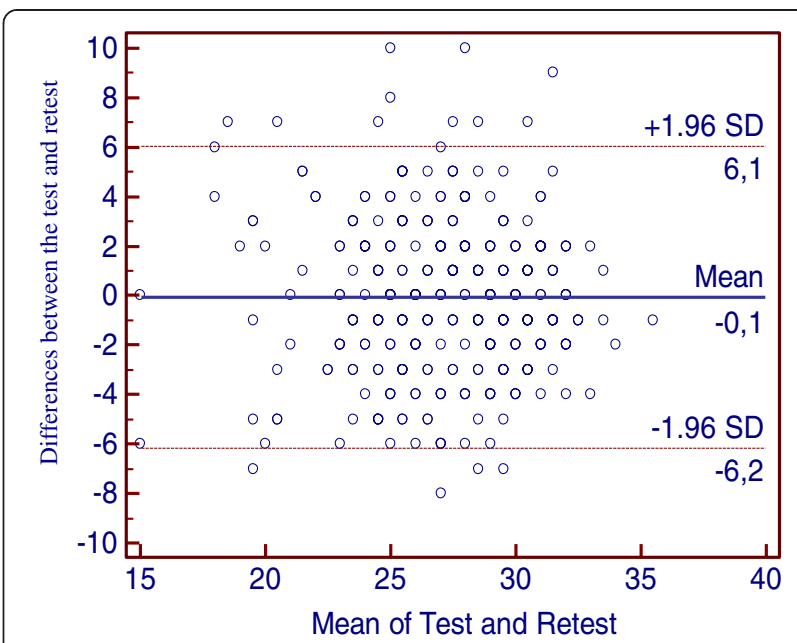

Figure 1 Bland-Altman plot of the eyes test-retest assessment $(n)=358$.

standard deviation was 3.63 , and the coefficient of repeatability was 6.24 .

\section{Discussion}

The primary purpose of the current study was to examine the long-term reliability of the Spanish translation of the Eyes test in Spain. To our knowledge, this is the first study providing evidence that the Eyes test is reliable and stable over a 1-year period in a nonclinical population sample. To determine the reliability of the Spanish version of the Eye test, we analyzed the distribution of responses for each item during testing and retesting one year later. The results indicate that not all items are equally difficult, which should increase the discriminant ability of the test. The distribution of difficulty across all items of the test was approximately normal and greater than $50 \%$ for the correct response. Despite the fact that less than $50 \%$ of the respondents correctly answered item 19, the majority did in fact choose the correct answer. In the Italian version of Eyes test similar percentages were obtained [26]. Further research should be conducted to determine if the item should be eliminated due to ambiguity or retained because it is difficult, and therefore useful in testing emotional discrimination.

Test-retest reliability using the ICC indicated a significant correlation between the total scores on the test and retest, demonstrating that results were stable over time. They also indicate that no learning occurred in the study population [34]. Item-by-item correlation analysis between test and retest showed that responses to all items except item 18 were stable over time. This finding implies that emotion recognition judgments, both correct and incorrect, persist over time. The relatively long interval of 1 year between test and retest further suggests that such persistence is not due to chance but to 
the existence of stable cognitive dispositions in recognizing complex emotions [1].

We used the graphical method of Bland-Altman to assess test-retest concordance on our Spanish translation of the Eyes test. This approach allowed us to analyze the position of test-retest differences relative to the testretest mean. This analysis showed that most responses on all items were concordant with one another; mean differences were 0 , and most differences fell within the $95 \%$ CI. The differences were homogeneous and appeared to be distributed randomly across all items of the test, with no evidence of a systematic bias or tendency. The small differences and their homogeneity lead us to conclude that the Eyes test is reliable and stable for up to 1 year, not only with respect to total scores but also to the distributions of answers for each item. These results may help guide the identification of items that discriminate between clinical and nonclinical populations in further studies.

This study is not without limitations. First, the proportion of women in our test population was much higher than that of men, raising the possibility of gender bias. Second, this study examined test-retest reliability over a relatively long period of 1 year. Future studies should also investigate the stability of the Spanish version over shorter time periods, since stability is expected to be greater over shorter periods [26].

Several studies using Eyes test have analyzed gender and age differences without conclusive results [26]. Our study did not address these issues. Future studies should investigate these differences and explore the mechanisms by which gender and age influence the development of theory of mind and emotional recognition. Additionally, it would be interesting to examine how other objective measures of emotion recognition, empathy, and emotional intelligence are related with Eyes test.

Numerous international studies using the Eyes test have shown group differences in emotion recognition and theory of mind between individuals diagnosed with schizophrenia $[7,8]$ or autism [9-11] and typical control groups. The Spanish version of the Eyes test will help in the diagnosis and effective implementation of intervention programs for individuals with impairment in social cognition in Spanish-speaking countries. This test will allow the comparison of an individual's score with the normative scores of Spanish samples and will enable researchers and clinicians to describe with accuracy any change of their scores before and after intervention programs.

\section{Conclusions}

In conclusion, the results from the current study suggest that the Eyes test is a reliable measure of theory of mind and recognition of complex emotions in adults, and that it is stable over a 1-year period in a nonclinical population. This Spanish version of the Eyes test will be useful in future research into social cognition in laboratory and clinical contexts, including cross-cultural and clinical investigations into autism and related neurodevelopmental conditions, in Spain and in other Spanish-speaking countries.

\section{Abbreviations \\ Cl: Confidence interval; ICC: Intraclass correlation coefficient; sd: Standard deviation.}

\section{Competing interests}

The authors declare they have no competing interests.

\section{Authors' contributions}

EGFA conceived of the study, participated in the data collection, analyzed the data and led preparation of the manuscript. RC and PFB conceived of the study and wrote the first draft of the manuscript. SBC contributed to writing the manuscript. All authors contributed to the interpretation of data helped to draft and revise the manuscript and have read and approved the final manuscript.

\section{Acknowledgements}

RC and PFB were supported in part by project SEJ-03036 from the Department of Economics, Science, and Business, Junta Andalucia (Spain). SBC was supported by the EU, the MRC, and the Wellcome Trust during the period of this work. He was part of the NIHR CLAHRC for Cambridgeshire and Peterborough NHS Foundation Trust.

\section{Author details}

${ }^{1}$ Department of Basic Psychology, Faculty of Psychology, The National Distance Education University (UNED), Madrid, Spain. ${ }^{2}$ Department of Developmental Psychology and Education, Faculty of Education Science, University of Huelva, Huelva, Spain. ${ }^{3}$ Department of Basic Psychology, Faculty of Psychology, University of Malaga, Campus Teatinos, s/n, 29071, Malaga, Spain. ${ }^{4}$ Autism Research Centre, Department of Psychiatry, Cambridge University, and CLASS Clinic, Cambridgeshire and Peterborough NHS Foundation Trust (CPFT), Cambridge, UK.

Received: 24 June 2013 Accepted: 27 August 2013

Published: 11 September 2013

\section{References}

1. Baron-Cohen S, Wheelwright S, Hill J, Raste Y, Plumb I: The "reading the mind in the eyes" test revised version: a study with normal adults, and adults with asperger syndrome or high-functioning autism. J Child Psychol Psychiatry 2001, 42:241-251.

2. Brothers $L$ : The neural basis of primate social communication. Motiv Emot 1990, 14:81-91.

3. Fiske ST, Taylor SE: Social cognition: From brains to culture. London, UK: SAGE Publications Limited; 2013.

4. Kunda Z: Social cognition: Making sense of people. Cambridge, MA: The MIT Press; 1999.

5. Baron-Cohen S: Mindblindness: An Essay on Autism and Theory of Mind. Cambridge, MA: MIT Press; 1995.

6. Pinkham AE, Penn DL, Perkins DO, Lieberman J: Implications for the neural basis of social cognition for the study of schizophrenia. Am J Psychiatry 2003, 160:815-824.

7. De Achával D, Costanzo EY, Villarreal M, Jáuregui IO, Chiodi A, Castro MN, Fahrer RD, Leiguarda RC, Chu EM, Guinjoan SM: Emotion processing and theory of mind in schizophrenia patients and their unaffected first-degree relatives. Neuropsychologia 2010, 48:1209-1215.

8. Green MF, Olivier B, Crawley JN, Penn DL, Silverstein S: Social cognition in schizophrenia: recommendations from the measurement and treatment research to improve cognition in schizophrenia new approaches conference. Schizophr Bull 2005, 31:882-887.

9. Baron-Cohen S: Essential Difference: Men, women and the extreme male brain. London, UK: Penguin; 2003 
10. Baron-Cohen S: Autism: the empathizing-systemizing (E-S) theory. Ann N Y Acad Sci 2009, 1156:68-80.

11. Lombardo MV, Chakrabarti B, Lai M-C, Baron-Cohen S: Self-referential and social cognition in a case of autism and agenesis of the corpus callosum. Mol Autism 2012, 3:14.

12. Adenzato M, Todisco P, Ardito RB: Social cognition in anorexia nervosa: evidence of preserved theory of mind and impaired emotional functioning. PloS one 2012, 7:e44414.

13. Harrison A, Tchanturia K, Treasure J: Attentional bias, emotion recognition, and emotion regulation in anorexia: state or trait? Biol Psychiatry 2010, 68:755-761.

14. Baron-Cohen S, Jaffa T, Davies S, Auyeung B, Allison C, Wheelwright S: Do girls with anorexia nervosa have elevated autistic traits? Mol Autism 2013, 4:24.

15. Derntl B, Seidel E-M, Kryspin-Exner I, Hasmann A, Dobmeier M: Facial emotion recognition in patients with bipolar I and bipolar II disorder. Br J Clinical Psychol 2009, 48:363-375.

16. Ryu V, An SK, Jo HH, Cho HS: Decreased P3 amplitudes elicited by negative facial emotion in manic patients: selective deficits in emotional processing. Neurosci Lett 2010, 481:92-96.

17. Machado-de-Sousa JP, Arrais KC, Alves NT, Chagas MHN, de Meneses-Gaya C, Crippa JADS, Hallak JEC: Facial affect processing in social anxiety: tasks and stimuli. J Neurosci Methods 2010, 193:1-6.

18. Frick C, Lang S, Kotchoubey B, Sieswerda S, Dinu-Biringer R, Berger M, Veser S, Essig M, Barnow S: Hypersensitivity in borderline personality disorder during mindreading. PLoS One 2012, 7:e41650.

19. Ihnen GH, Penn DL, Corrigan PW, Martin J: Social perception and social skill in schizophrenia. Psychiatry Res 1998, 80:275-286.

20. Rutherford MD, Baron-Cohen $S$, Wheelwright $S$ : Reading the mind in the voice: a study with normal adults and adults with asperger syndrome and high functioning autism. J Autism Dev Disord 2002, 32:189-194.

21. Scrimin S, Moscardino U, Capello F, Altoè G, Axia G: Recognition of facial expressions of mixed emotions in school-age children exposed to terrorism. Dev Psychol 2009, 45:1341-1352.

22. White $S$, Hill E, Happé F, Frith U: Revisiting the strange stories: revealing mentalizing impairments in autism. Child Dev 2009, 80:1097-1117.

23. Baron-Cohen S, Jolliffe T, Mortimore C, Robertson M: Another advanced test of theory of mind: evidence from very high functioning adults with autism or asperger syndrome. J Child Psychol Psychiatry 1997, 38:813-822.

24. Adams RB, Rule NO, Franklin RG, Wang E, Stevenson MT, Yoshikawa S, Nomura M, Sato W, Kveraga K, Ambady N: Cross-cultural reading the mind in the eyes: an fMRI investigation. J Cogn Neurosci 2010, 22:97-108.

25. Medina-Pradas C, Blas Navarro J, Alvarez-Moya EM, Grau A, Obiols JE: Emotional theory of mind in eating disorders. Int I Clin Heal Psychol 2012, 12:189-202.

26. Vellante M, Baron-Cohen S, Melis M, Marrone M, Petretto DR, Masala C, Preti A: The "reading the mind in the eyes" test: systematic review of psychometric properties and a validation study in italy. Cogn Neuropsychiatry 2012, 18:326-354.

27. Bora E, Vahip S, Gonul AS, Akdeniz F, Alkan M, Ogut M, Eryavuz A: Evidence for theory of mind deficits in euthymic patients with bipolar disorder. Acta Psychiatr Scand 2005, 112:110-116.

28. Havet-Thomassin V, Allain P, Etcharry-Bouyx F, Le Gall D: What about theory of mind after severe brain injury? Brain Inj 2006, 20:83-91.

29. Kelemen O, Kéri S, Must A, Benedek G, Janka Z: No evidence for impaired "theory of mind" in unaffected first-degree relatives of schizophrenia patients. Acta Psychiatr Scand 2004, 110:146-149.

30. Kunihira Y, Senju A, Dairoku H, Wakabayashi A, Hasegawa T: "Autistic" traits in non-autistic Japanese populations: relationships with personality traits and cognitive ability. J Autism Dev Disord 2006, 36:553-566.

31. Voracek M, Dressler SG: Lack of correlation between digit ratio (2D:4D) and Baron-Cohen's "reading the mind in the eyes" test, empathy, systemising, and autism-spectrum quotients in a general population sample. Personal Individ Differ 2006, 41:1481-1491.

32. Bänziger $T$, Scherer KR, Hall JA, Rosenthal R: Introducing the MiniPONS: a short multichannel version of the profile of nonverbal sensitivity (PONS). J Nonverbal Behav 2011, 35:189-204.
33. Yildirim EA, KaŞar M, Güdük M, Ateş E, Küçükparlak I, Özalmete EO: Investigation of the reliability of the "reading the mind in the eyes test" in a turkish population. Turkish J Psychiatry 2011, 22:177-186.

34. Hallerbäck MU, Lugnegård T, Hjärthag F, Gillberg C: The reading the mind in the eyes test: test-retest reliability of a Swedish version. Cogn Neuropsychiatry 2009, 14:127-143.

35. Bland JM, Altman DG: Statistical methods for assessing agreement between two methods of clinical measurement. Lancet 1986, 1:307-310,

doi:10.1186/2040-2392-4-33

Cite this article as: Fernández-Abascal et al:: Test-retest reliability of the 'Reading the Mind in the Eyes' test: a one-year follow-up study.

Molecular Autism 2013 4:33.

\section{Submit your next manuscript to BioMed Central and take full advantage of:}

- Convenient online submission

- Thorough peer review

- No space constraints or color figure charges

- Immediate publication on acceptance

- Inclusion in PubMed, CAS, Scopus and Google Scholar

- Research which is freely available for redistribution

Submit your manuscript at www.biomedcentral.com/submit
() Biomed Central 